\title{
Resveratrol from Red Grapes - Pedestrian Polyphenol or Useful Anticancer Agent?
}

Author

Affiliation
Andreas J. Gescher

Cancer Biomarkers and Prevention Group, Department of Cancer Studies and Molecular Medicine, University of Leicester, Leicester, U.K.
Key words

- Resveratrol

- cancer chemoprevention

- caloric restriction

- analogues received November 19, 2007

revised March 5, 2008

accepted March 11, 2008

Bibliography

Dol 10.1055/s-2008-1074516

Planta Med 2008; 74: 1651-

1655

(c) Georg Thieme Verlag KG

Stuttgart · New York

Published online April 29, 2008

ISSN 0032-0943

Correspondence

\section{Andreas J. Gescher}

Cancer Biomarkers and

Prevention Group

Department of Cancer Studies

and Molecular Medicine

University of Leicester

Leicester LE2 7LX

U.K.

Tel.: +44-116-223-1856

Fax: +44-116-223-1855

ag15@le.ac.uk

\section{Abstract \\ $\nabla$}

Resveratrol is a phytoalexin with cancer chemopreventive properties in preclinical models of carcinogenesis. The mechanisms via which resveratrol is thought to exert chemopreventive efficacy are inhibition of cyclooxygenase enzymes, inhibition of angiogenesis, modulation of drug metabolising enzymes, antioxidation and alterations of cell cycle components and apoptotic machinery. Pharmacokinetic evidence in rodents and humans suggests that the bioavailability of resveratrol is very low and that resveratrol conju- gates are the major circulating agent-derived species. The recent realisation that resveratrol can mimic caloric restriction in several species has generated a lot of interest. Attempts to design analogues with the aim of optimising resveratrol pharmacology have furnished stilbenes with different aryl subtituents, e.g., methoxy instead of hydroxy. Some of these derivatives possess more potent pharmacological properties than the lead compound. More work is required to elucidate the role of metabolites in the pharmacological activity of resveratrol.

\section{Introduction}

\section{$\nabla$}

Phytochemicals with beneficial effects on health regularly make newspaper headlines. Media awareness reflects the considerable interest of the general public in naturally occurring remedies rooted in the assumptions that the plant kingdom harbours remedies against all sorts of ills, and that many of these remedies have still to be discovered. The realisation that fruits and vegetables can prevent diseases such as cancer has engendered an intense search for the constituents which may be responsible for these properties. Resveratrol (3,5,4'-trihydroxy-trans-stilbene, 1 in - Fig. 1) is an example of a dietary constituent which has been shown over the last decade to possess a fascinating spectrum of pharmacological properties. Resveratrol was first isolated from the roots of white hellebore (Vertarum grandiflorum O. Loes) in 1940 and later from the roots of Polygonum cuspidatum, a plant used in Chinese and Japanese medicine. In 1992, resveratrol was suggested to explain some of the cardioprotective effects of red wine [1]. Since then many reports have shown that resveratrol can prevent, or delay, the progression of illnesses such as cancer, cardiovascular disease and is- chaemic injury, as well as enhance stress resistance and extend the lifespan of various organisms from yeast to vertebrates. In 1997 Jang et al. [2] published a paper in which the ability of resveratrol to inhibit diverse cellular events associated with initiation, promotion and progression of cancer was described. This paper fired the imagination of the cancer chemoprevention research community. During the 11 years since its publication, literally hundreds of reports have appeared in the literature in which numerous cellular and biochemical mechanisms of resveratrol and their potential role in its putative cancer chemopreventive activity have been discussed. As a triphenol, resveratrol is exceedingly prone to undergo metabolic conjugation, so that its bioavailability is extremely low. Its intriguing efficacy in spite of low bioavailability constitutes a conundrum [3]. This perspective aims at exploring whether recent results of investigations of resveratrol, especially those in humans, help resolve the conundrum. It focuses in particular on the mechanisms and pharmacokinetics of resveratrol. It also describes analogues which have been synthesized with the aim to optimise pharmacological features of the parent agent. There have been several incisive and comprehensive re- 
views of the health effects and pharmacokinetics of resveratrol [4], [5], [6], yet information of the pharmacology of resveratrol in humans remains scarce.

\section{How can Resveratrol Prevent Cancer?}

$\nabla$

Prominent among the mechanisms, which may mediate the cancer chemopreventive efficacy of resveratrol, are inhibition of cyclooxygenase enzymes, inhibition of angiogenesis, modulation of drug metabolising enzymes, antioxidation and alterations in cell cycle and apoptotic machinery. Jang et al. originally proposed that resveratrol inhibits the enzymatic activity of both cylooxygenase enzymes COX-1 and -2 [2]. Epidemiological evidence suggests that long-term inhibition of cyclooxygenase significantly reduces the risk of developing many cancers, and deletion of the gene that encodes COX-2 is protective in a mouse model of colorectal cancer [7]. Resveratrol inhibited COX-1 in a somewhat selective fashion and reduced COX-2 at the mRNA level [8], [9], [10], [11]. Resveratrol, delivered systemically, inhibited the tumour-induced neovascularisation required to support solid tumour growth, illustrating its anti-angiogenic properties [12], [13]. Resveratrol can modulate the expression and activity of multiple drug-metabolising enzymes. It inhibited various cytochrome P450 s [14], [15], [16], [17] and induced expression of phase II drug detoxifying enzymes [18]. These activities suggest that resveratrol may reduce the exposure of cells to carcinogens by decreasing carcinogen formation and/or increasing their detoxification. Reactive oxygen species (ROS) have been shown to be involved with the initiation and progression of cancer via damaging DNA and other biomolecules. Resveratrol has antioxidant capacity, as reflected by its ability to increase plasma antioxidant activity and to decrease lipid peroxidation [19], [20], [21]. Resveratrol possesses also anti-proliferative and proapoptotic effects in tumour cell lines [22]. These effects may be mechanistically linked to its abilities to down-regulate cell cycle proteins [23], [3], [25] and increase apoptosis [26], [27], [28] in tumour models in vivo. Resveratrol sensitised tumour cells to apoptosis induced by TRAIL (tumour necrosis factor-related apoptosis-inducing ligand) [29], and this property may explain its pro-apoptotic effects in vivo.

At the end of 2006, two reports suggested that resveratrol is a caloric restriction mimetic in lower organisms and mice, associated with its ability to activate sirtuin proteins and to extend life-span [30], [31]. Sirtuins are NAD-dependent deacetylases involved in gene silencing processes germane to aging, blockade of apoptosis and promotion of cell survival. How this mechanism of resveratrol may contribute to explain its chemopreventive properties is unclear and currently the focus of intense investigation.
Paradoxically, resveratrol can counteract radical oxygen species (ROS) production, induce apoptosis and inhibit cell growth, but it can also engage sirtuins to increase mitochondrial metabolism and, in turn, ROS production, thus enhancing cell survival. Consistent with this confusing notion, resveratrol at low concentrations ( $4-8 \mu \mathrm{M}$ ) had a pro-oxidant effect in human-derived leukaemia cells, and it inhibited events associated with apoptosis induced by hydrogen peroxide and the anticancer drugs vincristine and daunorubicin [32]. This finding hints at the possibility that under certain conditions resveratrol in combination with cytotoxicants may counteract rather than augment their therapeutic efficacy.

Scanning internet websites devoted to life enhancement shows that a surprisingly large number of healthy humans now ingest resveratrol at high doses with the objective to prolong their lives, without bearing in mind that the safety of high-dose resveratrol ingested for a long term is unknown.

\section{Pharmacokinetics of Resveratrol $\nabla$}

In rodents resveratrol possesses a short initial half-life, 8$14 \mathrm{~min}$ [33], [34] as it is metabolised extensively and rapidly. Results from clinical trials are essentially compatible with the rodent studies. Table 1 describes the widely varying situations in which resveratrol has been studied in humans, administered either as the pure compound [35], [36] or as a constituent of wine or grapefruit juice or grape extract [37], [38], [39], [40], [41], [42]. Walle et al. [35] showed that the bulk of an i.v. dose of $25 \mathrm{mg}$ per human resveratrol was converted to sulphate conjugates within $30 \mathrm{~min}$. Serum peak levels of resveratrol were $<22$ nM. Five metabolites were identified in the urine, resveratrol monosulphate, two isomeric monoglucuronides, dihydroresveratrol monosulphate and dihydroresveratrol monoglucuronide. Total sulphate and glucuronide conjugates accounted for $\sim 37$ and $19 \%$, respectively, of the metabolites in the urine, and there were only trace amounts of free resveratrol. Recently, a study of the pharmacokinetics of oral resveratrol administered at single high doses $(0.5,1,2.5$ or $5 \mathrm{~g})$ was conducted in healthy human volunteers [36]. These doses were safe, and resveratrol and six metabolites were recovered from plasma and urine. Peak plasma level of resveratrol at the highest dose $(5 \mathrm{~g})$ was $2.4 \mu \mathrm{M}$, which occurred $1.5 \mathrm{~h}$ post-dose. Peak levels of two monoglucuronides and resveratrol 3-sulphate were 3- to 8-fold higher. The area under the plasma concentration curve values for resveratrol 3-sulphate and resveratrol monoglucuronides were up to 23 times greater than those of resveratrol. Urinary excretion of resveratrol and its metabolites was rapid, with $77 \%$ of all urinary agent-derived species excreted within $4 \mathrm{~h}$ after the lowest dose $(0.5 \mathrm{~g})$.

Table 1 Clinical trials of resveratrol

\begin{tabular}{|ll}
\hline Study cohort (n) & Intervention \\
\hline Healthy volunteers (44) & Lyophilized grape powder \\
\hline Healthy volunteers (6) & Resveratrol in whisky/grape juice \\
\hline Healthy volunteers (25) & Red wine \\
\hline Healthy volunteers (12) & Red wine, fruit or vegetable juice \\
\hline Healthy volunteers (20) & Red wine \\
\hline Coronary heart disease patients (30) & Grape extract \\
\hline Healthy volunteers (6) & Pure resveratrol \\
\hline Healthy volunteers (40) & Pure resveratrol \\
\hline
\end{tabular}


Conjugate formation typically reduces cell permeability and aids drug excretion, therefore the reported in vivo efficacy of resveratrol in spite of its low bioavailability has led to speculation that its metabolites could mediate, at least in part, the efficacy exerted by the parent molecule. Concentrations of resveratrol in red wine vary widely, but a reasonable estimate is about $5 \mathrm{mg}$.L-1 $(22 \mu \mathrm{M})$ [43]. Assuming a consistent daily intake of two glasses of wine $(-375 \mathrm{~mL})$ a person weighing $70 \mathrm{~kg}$ would receive a dose of $\sim 27 \mu \mathrm{g}$ per kg body weight each day. The plasma concentrations after such a low dose are clearly vanishingly small, in the 1 - 10 nM range [39], certainly orders of magnitudes below those found to elicit most pharmacological effects reported in vitro. Therefore, it seems prudent to explore the pharmacology of resveratrol from two separate standpoints, firstly considering it as diet constituent, and secondly as agent in its own right which just happens to be diet-derived. Russo [44] surmised that the diet constituent resveratrol, i.e., resveratrol at low doses, may be useful as a caloric restriction mimic, whilst at high doses, it may be a promising chemotherapeutic agent, but in neither case useful as a cancer chemopreventive agent. This interpretation is based on the findings that low doses generate concentrations capable of mimicking caloric restriction, but insufficient to engage anti-carcinogenesis, whilst high doses, which are pharmaceutically unfeasible in the chemoprevention paradigm, engender levels exerting anti-proliferative and proaptototic effects. Nevertheless, in the light of the polymechanistic nature of the effect of resveratrol, its seems premature to write off its potential chemopreventive activity. This conclusion seems especially pertinent considering the possibility that the caloric restriction-mimetic mechanisms which resveratrol engages may contribute to anti-carcinogenicity. It is worth bearing in mind that whilst most anti-carcinogenic mechanisms reported for resveratrol in cells in vitro require concentrations of more than 10-5 M, it can elicit some survival-inhibitory biochemical events at concentrations approaching those achieved in vivo (reviewed in [45]).

The maximum tolerated dose of resveratrol has not been properly established, but at $300 \mathrm{mg} / \mathrm{kg}$ it did not have detrimental effects in the rat [46].

\section{Resveratrol Analogues}

$\nabla$

Not surpisingly the diverse attractive pharmacological features of resveratrol in concert with its relatively simple chemical structure have inspired medicinal chemists to synthesise novel analogues with cancer chemopreventive/chemotherapeutic efficacy superior to that of parent resveratrol. Efforts have focussed on structure-activity relationships of substituted stilbene derivatives to optimise cytotoxic potency, ability to inhibit activation of NFkB, tyrosine kinase or cytochrome P450 or to exert antioxidation [47], [48], [49], [50], [51]. For example a series of cis- and trans-stilbenes related to resveratrol with varying aryl, hydroxy, methoxy and/or amine moieties has been synthesised and evaluated for ability to induce apoptosis in human leukaemia-derived HL60 cells [52]. Among the analogues cis-3,4',5-trimethoxy-3'-aminostilbene and cis-3,4',5-trimethoxy-3'-hydroxystilbene (2, 3, $\odot$ Fig. 1) were found to possess potency superior to that of the lead compound resveratrol. Other workers have generated polyhydroxystilbenes, exemplified by 3,3',4',5-tetrahydroxystilbene (4, $\odot$ Fig. $\mathbf{1}$ ), to maximise the antioxidant activity of this type of molecule [53]. A relatively subtle chemical change brought about by methylation of the stilbene hydroxy moieties increased several pharmacological potencies of resveratrol. This increased potency may be, at least in part, due to the fact that methoxy moieties tend to be less vulnerable to attack by metabolising enzymes than hydroxyl groups. Pertinent in this context is the finding that pterostilbene, a bis-methylated resveratrol, found in blueberries (5, $\odot$ Fig. $\mathbf{1}$ ) at $40 \mathrm{ppm}$ in the diet potently suppressed aberrant crypt foci formation in the azoxymethane-induced colon carcinogenesis rat model and suppressed inducible nitric oxide synthase expression [54].

The recent realisation that resveratrol may extend life-span, and the assumption that this property may ultimately be observed in humans, has engendered a flurry of medicinal chemistry projects in the pharmaceutical industry. These activities are primarily aimed at generating novel patentable molecules which activate sirtuins, possess life-extending properties and mimic health effects of calorie restriction without requiring a change in eating habits, undoubtedly an attractive endeavour<smiles>Oc1ccc(/C=C/c2cc(O)cc(O)c2)cc1</smiles><smiles>COc1cc(/C=C/c2ccc(OC)c(N)c2)cc(OC)c1</smiles>
2<smiles>COc1cc(/C=C/c2ccc(OC)c(O)c2)cc(OC)c1</smiles><smiles>Oc1cc(O)cc(C=Cc2ccc(O)c(O)c2)c1</smiles>

Fig. 1 Structures of resveratrol (1) and resveratro analogues discussed in the text, cis-3,4,5-trimethoxy-3'-aminostilbene (2), cis-3,4',5-trimethoxy3'-hydroxystilbene (3), 3,3',4',5-tetrahydroxystilbene (4) and pterostilbene (5). 
from a financial standpoint. It seems worthwhile to test these analogues, when they will be available, for cancer chemopreventive properties.

\section{Future Work}

$\nabla$

Are we any closer to resolving the conundrum posed by the undeniable efficacy of resveratrol in preclinical models in spite of its low systemic availability? Hardly. It is still unclear whether metabolites contribute to resveratrol efficacy. Experiments to explore this issue are afoot. The differential effects of dietary (i.e., low) doses of resveratrol on the one hand and pharmacological (i.e., high) doses on the other has sparked current investigations of dose-dependent differences in metabolite profiles obtained after consumption of either red wine or purified resveratrol. Results from studies of this type will undoubtedly help explain dose-response issues related to resveratrol pharmacology. Further chemical synthesis of resveratrol cogeners might generate molecules with biological activities superior to those of resveratrol. It remains to be seen whether new synthetic caloric restriction mimetics modelled on resveratrol will prolong human life and whether such molecules exert cancer chemopreventive activity. Whilst studies in humans conducted hitherto support the tentative notion that resveratrol is a safe molecule, it will take a long time to establish whether chemically designed resveratrol analogues retain the safety of the parent molecule. Lack of knowledge of their safety will for a long time confound consideration of such novel molecules as potential cancer chemopreventive agents. The findings briefly highlighted here show that resveratrol is certainly not a pedestrian polyphenol. On the other hand, it is unlikely to turn out to be a miraculous anticancer remedy, even though it constitutes undoubtedly a fascinating molecule which will intrigue cancer pharmacologists for a considerable time to come.

\section{References}

1 Siemann EH, Creasy LL. Concentration of the phytoalexin resveratrol in wine. Am J Eno Vitic 1992; 43: 49-52

2 Jang M, Cai L, Udeani GO, Slowing KV, Thomas CF, Beecher CW et al. Cancer chemopreventive activity of resveratrol, a natural product derived from grapes. Science 1997; 275: 218-20

3 Gescher AJ, Steward WP. Relationship between mechanisms, bioavailibility, and preclinical chemopreventive efficacy of resveratrol: A conundrum. Cancer Epidemiol Biomarkers Prev 2003; 12: 953 - 7

4 Baur JA, Sinclair DA. Therapeutic potential of resveratrol: the in vivo evidence. Nat Rev Drug Discov 2006; 5: 493 -506

5 Wenzel E, Somoza V. Metabolism and bioavailability of trans-resveratrol. Mol Nutr Food Res 2005; 49: $472-81$

6 Aggarwal BB, Shishodia S. Resveratrol in health and disease Boca Raton, FL: CRC Press, Taylor and Francis Group; 2006

7 Oshima M, Dinchuk JE, Kargman SL, Oshima H, Hancock B, Kwong E et al. Suppression of intestinal polyposis in Apc $\delta 716$ knockout mice by inhibition of cyclooxygenase 2 (COX-2). Cell 1996; 87: 803-9

8 Li ZG, Hong T, Shimada Y, Komoto I, Kawabe A, Ding Y et al. Suppression of $\mathrm{N}$-nitroso-methylbenzylamine (NMBA)-induced esophagael tumourigenesis in F344 rats by resveratrol. Carcinogenesis 2002; 23: $1531-6$

9 Aziz M, Afaq F, Ahmad N. Prevention of ultraviolet-B radiation damage by resveratrol in mouse skin is mediated via modulation in survivin. Photochem Photobiol 2004; 81: 25 - 31

10 Subbaramaiah $K$, Chung WJ, Michaluart P, Telang N, Tanabe T, Inoue $H$ et al. Resveratrol inhibits cyclooxygenase-2 transcription and activity in phorbol ester-treated human mammary epithelial cells. J Biol Chem 1998; 273: $21875-82$

11 Martin AR, Villegas I, La Casa C, de la Lastra CA. Resveratrol, a polyphenol found in grapes, suppresses oxidative damage and stimulates apoptosis during early colonic inflammation in rats. Biochem Pharmacol 2004; 67: 1399-1410

12 Kimura Y, Okuda H. Resveratrol isolated from Polygonuum cuspidatum root prevents tumor growth and metastasis to lung and tumor-induced neovascularisation in Lewis lung carcinoma-bearing mice. J Nutr 2001; 131: $1844-9$

13 Tseng SH, Lin SM, Chen JC, Su YH, Huang HY, Chen CK et al. Resveratrol suppresses the angiogenesis and tumor growth of gliomas in rats. Clin Cancer Res 2004; 10: 2190-2

$14 \mathrm{Yu}$ C, Shin YG, Kosmeder JW, Pezzuto JM, van Breemen RB. Liquid chromatography/tandem mass spectrometric determination of inhibition of human cytochrome P450 isozymes by resveratrol and resveratrol3-sulfate. Rapid Commun Mass Spectrom 2003; 17: 307-13

15 Piver B, Berthou F, Dreano Y, Lucas D. Inhibition of CYP3A, CYP1A and CYP2E1 activities by resveratrol and other non volatile red wine components. Toxicol Lett 2001; 125: 83-91

16 Chang TK, Lee WB, Ko HH. Trans-resveratrol modulates the catalytic activity and mRNA expression of the procarcinogen-activating human cytochrome P450 1B1. Can J Physiol Pharmacol 2000; 78: 874 - 81

17 Chan WK, Delucchi $A B$. Resveratrol, a red wine constituent, is a mechanism-based inactivator of cytochrome P450 3A4. Life Sci 2000; 67: $3103-12$

18 Cao Z, Li Y. Potent induction of cellular antioxidants and phase 2 enzymes by resveratrol in cardiomyocytes: protection against oxidative and electrophilic injury. Eur J Pharmacol 2004; 489: 39-48

19 Sengottuvelan M, Viswanathan P, Nalini N. Chemopreventive effect of trans-resveratrol - a phytoalexin against colonic aberrant crypt foci and cell proliferation in 1,2-dimethylhydrazine induced colon carcinogenesis. Carcinogenesis 2005; 27: 1038 - 46

20 Miura D, Miura Y, Yagasaki K. Hypolipidemic action of dietary resveratrol, a phytoalexin in grapes and red wine, in hepatoma-bearing rats. Life Sci 2003; 73: $1393-400$

21 Wenzel E, Soldo T, Erbersdobler H, Somoza V. Bioactivity and metabolism of trans-resveratrol orally administered to Wistar rats. Mol Nutr Food Res 2005; 49: 482 - 94

22 Clement MV, Hirpara JL, Chawdhury SH, Pervaiz S. The chemopreventive agent resveratrol, a natural product derived from grapes, triggers CD95 signaling-dependent apoptosis in human tumor cells. Blood 1998; 92: $996-1002$

$23 \mathrm{Yu}$ L, Sun ZJ, Wu SK, Pan CE. Effect of resveratrol on cell cycle proteins in murine transplantable liver cancer. World J Gastroenterol 2003; 9: $2341-3$

24 Schneider Y, Duranton B, Gosse F, Schleiffer R, Seiler N, Raul F. Resveratrol inhibits intestinal tumourigenesis and modulates host-defenserelated gene expression in an animal model of human familial adenomatous polyposis. Nutr Cancer 2001; 39: $102-7$

25 Reagan-Shaw S, Afaq F, Aziz MH, Ahmad N. Modulation of critical cell cycle regulatory events during chemoprevention of ultraviolet Bmediated responses by resveratrol in SKH-1 hairless mouse skin. Oncogene 2004; 23: 5151 - 60

26 Garvin S, Ollinger K, Dabrosin C. Resveratrol induces apoptosis and inhibits angiogenesis in human breast cancer xenografts in vivo. Cancer Lett 2006; 231: $113-22$

27 Provinciali M, Re F, Donnini A, Orlando F, Bartozzi B, Di Stasio Get al. Effect of resveratrol on the development of spontaneous mammary tumors in HER-2/neu transgenic mice. Int J Cancer 2005; 115: 36 - 45

28 Zhou HB, Chen JJ, Wang WX, Cai JT, Du Q. Anticancer activity of resveratrol on implanted human primary gastric carcinoma cells in nude mice. World J Gastroenterol 2005; 11: 280 - 4

29 Fulda S, Debatin KM. Sensitization for tumor necrosis factor-related apoptosis-inducing ligand-induced apoptosis by the chemopreventive agent resveratrol. Cancer Res 2004; 64: 337-46

30 Baur JA, Pearson KJ, Price NL, Jamieson H A, Lerin C, Kalra A et al. Resveratrol improves health and survival of mice on a high-calorie diet. Nature 2006; 444: 337-42

31 Lagouge M, Argmann C, Gerhart-Hines Z, Meziane H, Lerin C, Daussin F et al. Resveratrol improves mitochondrial function and protects against metabolic disease by activating SIRT1 and PGC-1 alpha. Cell 2006; 127: 1109-22

32 Ahmad KA, Clement MV, Hanif IM, Pervaiz S. Resveratrol inhibits druginduced apoptosis in human leukemia cells by creating an intracellular milieu non-permissive for death execution. Cancer Res 2004; 64: $1452-9$

33 Marier JF, Vachon P, Gritsas A, Zhang J, Moreau JP, Ducharme MP. Metabolism and disposition of resveratrol in rats: extent of absorption, 
glucuronidation, and enterohepatic recirculation evidenced by a linked-rat model. J Pharmacol Exp Ther 2002; 320: 369- 73

34 Asensi M, Medina I, Ortega A, Carretero J, Bano MC, Obrador E et al. Inhibition of cancer growth by resveratrol is related to its low bioavailability. Free Radic Biol Med 2002; 33: 387-98

35 Walle T, Hsieh F, DeLegge MH, Oatis JE, Walle UK. High absorption but very low bioavailability of oral resveratrol in humans. Drug Metab Disp 2004; 32: 1377-82

36 Boocock DJ, Faust GES, Patel KR, Schinas AM, Brown VA, Ducharme MP et al. Phase I dose escalation pharmacokinetic study in healthy volunteers of resveratrol, a potential cancer chemopreventive agent. Cancer Epidemiol Biomarkers Prev 2007; 16: 1246 - 52

37 Meng XF, Maliakal P, Lu H, Lee MJ, Yang CS. Urinary and plasma levels of resveratrol and quercetin in humans, mice, and rats after ingestion of pure compounds and grape juice. J Agric Food Chem 2004; 52: 935- 42

38 Zern TL, Wood RJ, Greene C, West KL, Liu YZ, Aggarwal D et al. Grape polyphenols exert a cardioprotective effect in pre- and postmenopausal women by lowering plasma lipids and reducing oxidative stress. J Nutr 2005; 135: $1911-7$

39 Vitaglione P, Sforza S, Galaverna G, Ghidini C, Caporaso N, Vescovi PP et al. Bioavailability of trans-resveratrol from red wine in humans. Mol Nutr Food Res 2005; 49: 495 - 504

40 Goldberg DA, Yan J, Soleas GJ. Absorption of three wine-related polyphenols in three different matrices by healthy subjects. Clin Biochem 2003; 36: 79-87

41 Zamora-Ros $R$, Urpi-Sarda $M$, Lamuela-Raventos $R M$, Estruch $R$, Vazquez-Agell M, Serrano-Martinez $M$ et al. Diagnostic performance of urinary resveratrol metabolites as a biomarker of moderate wine consumption. Clin Chem 2006; 52: $1373-80$

42 Lekakis J, Rallidis LS, Andreadou I, Vamvakou G, Kazantzoglou G, Magiatis $P$ et al. Polyphenolic compunds from red grapes acutely improve endothelial function in patients with coronary heart disease. Eur J Cardiovasc Prev Rehabil 2005; 12: 596-600

43 Pervaiz S. Resveratrol: from grapevines to mammalian biology. FASEB J 2003; 17: 1975-85
44 Russo GL. Ins and outs of dietary phytochemicals in cancer chemoprevention. Biochem Pharmacol 2007; 74: $533-44$

45 Howells LM, Moiseeva EP, Neal CP, Foreman BE, Kandreadi CK, Sun YY et al. Predicting the physiological relevance of in vitro cancer preventive activities of phytochemicals. Acta Pharmacol Sin 2007; 28: 1274 - 304

46 Crowell JA, Korytko PJ, Morrissey RL, Booth TD, Levine BS. Resveratrolassociated renal toxicity. Toxicol Sci 2004; 82: $614-9$

47 Pettit GR, Grealish MP, Jung MK, Hamel E, Pettit RK, Chapuis JC et al. Antineoplastic agents. 465. Structural modification of resveratrol: sodium resveratrin phosphate. J Med Chem 2002; 45: 2534-42

48 Kim S, Ko H, Park JE, Jung S, Lee SK, Chun YJ. Design, synthesis and discovery of novel trans-stilbene analogues as potent and selective human cytochrome P450 1B1 inhibitors. J Med Chem 2002; 45: 160 - 4

49 Thakkar K, Geahlen RL, Cushman M. Synthesis and protein-tyrosine kinase inhibitory activity of polyhydroxylated stilbene analogues of piceatannol. J Med Chem 1993; 36: 2950-5

50 Cushman M, Nagarathnam D, Gopal D, He HM, Lin CM, Hamel E. Synthesis and evaluation of analogues of (Z)-1-(4-methoxyphenyl)-2(3,4,5-trimethoxyphenyl)ethene as potential cytotoxic and antimitotic agents. J Med Chem 1992; 35: 2293-306

51 Heynekamp IJ, Weber WM, Hunsaker LA, Gonzales AM, Orlando RA, Deck $L M$ et al. Substituted trans-stilbenes, including analogues of the natural product resveratrol, inhibit the human tumor necrosis factor alpha-induced activation of transcription factor nuclear factor kappaB. J Med Chem 2006; 49: 7182 -9

52 Roberti M, Pizzirani D, Simoni D, Rondanin R, Baruchello R, Bonora C et al. Synthesis and biological evaluation of resveratrol and analogues as apoptosis-inducing agents. J Med Chem 2003; 46: 3546- 54

53 Murias M, Jager W, Handler N, Erker T, Horvath Z, Szekeres T et al. Antioxidant prooxidant and cytotoxic activity of hydroxylated resveratrol analogues: structure-activity relationship. Biochem Pharmacol 2005; 69: $903-12$

54 Suh N, Paul S, Hao XP, Simi B, Xiao H, Rimando AM et al. Pterostilbene, an active constituent of blueberries, suppresses aberrant crypt foci formation in the azoxymethane-induced colon carcinogenesis model in rats. Clin Cancer Res 2007; 13: 350-5 